\title{
Sensitivity Study of the 5-station configuration of ARA
}

\author{
Simon Archambault ${ }^{1, \star}$, for the ARA collaboration \\ ${ }^{1}$ Chiba University
}

\begin{abstract}
The Askaryan Radio Array (ARA) is an experiment looking for the Askaryan emission of cosmogenic neutrinos interacting in the Antarctic ice. During the last Antarctic summer, two new stations have been added to the experiment, as well as a prototype version of a phased array, attached to one of the new stations. With these stations, ARA sensitivity should become comparable to IceCube's from $10^{18} \mathrm{eV}$ and above. To confirm this, the sensitivity is going to be calculated through new simulations developed in the IceCube framework, with an antenna model built from in-situ calibration measurements.
\end{abstract}

\section{Introduction}
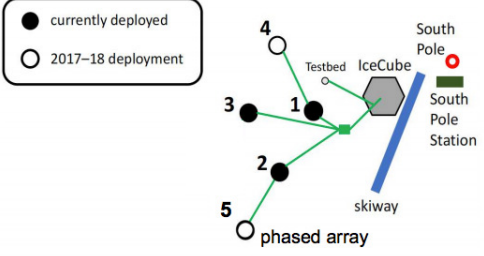

$\stackrel{2 \mathrm{~km}}{\longrightarrow}$

Figure 1. Layout of the current ARA array, comprised of 5 stations. Also shown are the locations of IceCube, the skiway and the South Pole station.

The Askaryan Radio Array (ARA) is an experiment located at the South Pole, aiming to detect comosgenic neutrinos, neutrinos coming from ultra-high-energy cosmic rays (UHECRs) that interact with the cosmic microwave background [1-3]. These neutrinos would interact in the Antarctic ice sheet, generating a radio signal known as Askaryan emissions, that can be picked up by the experiment $[4,5]$.

As of January 2018, ARA now has 5 stations deployed, as shown Figure 1. Each station is separated by $2 \mathrm{~km}$ spacing, to maximize the experiment's effective area at $10^{18} \mathrm{eV}$ [6]. This configuration should, in principle, make ARA as sensitive as the current IceCube configuration at $10^{18} \mathrm{eV}$. A new simulation package for ARA is being developed, using the IceCube framework, in order to confirm this expectation. For this simulation package to work, it is important to properly describe the behaviour of the antennas. For this purpose, in-situ measurements were performed at the South Pole, to help characterizing the realized gain of the antennas as a function of frequency and hit angle.

\section{Understanding Antennas}

ARA uses 2 different types of antennas, one for each polarization (vertical polarization, or VPol, and horizontal polarization, HPol). These are useful to identify the polarization of the incoming signal, which helps in reconstructing the original arrival direction of the neutrino itself.

^e-mail: sarchambault@icecube.wisc.edu 
Figure 2 shows a model of each antenna. Their design was constrained by the need to fit in the holes dug in the Antarctic ice, hence a cylindrical design $15 \mathrm{~cm}$ wide. To catch the Askaryan signal, they need to be broadband, for a range of frequencies of 150-800 MHz. Finally, their sensitivity needs to be azimuthally-symmetric. VPol antennas are birdcage dipoles, while HPol antennas are slotted copper cylinders, loaded with internal ferrites.

\section{In-Situ Calibration}

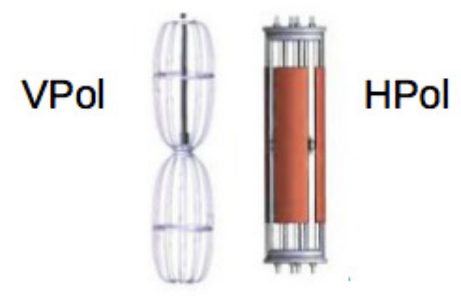

Figure 2. Models of the VPol and HPol antenna designs used by ARA.

Each ARA station is equipped with 2 calibration strings, approximately 40 meters away from the center of the station. Each string has a HPol and a VPol antenna, and a pulser is connected to them and excites them as needed to send a calibration signal to the other strings. To measure the responses of the VPol antenna, the calibration pulser (calpulser) sends a signal to the VPol antenna on its string, and similarly for the HPol antennas. These signals can then be used to calculate the realized gain of the antennas as well as the signal chain gain. Figure 3 shows a schematic of this measurement.

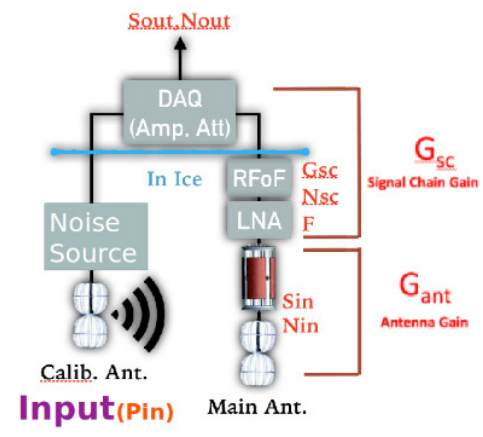

Figure 3. Schematic of the In-Situ antenna gain measurement. The different components in the calibration string as well as in a typical ARA string is represented.

In the summer of 2017, a special calibration measurement was performed on one of the stations, where the calibration string was progressively raised from its nominal position. Taking measurements at different positions allowed to measure the antennas' response at different zenith angles, giving a fuller picture of their behavior. The right plot of Figure 4 shows the resulting angular response of the VPol antennas.

\section{Antenna Model}

This measurement can be used to develop an antenna model, useful for simulations. Figure 4 shows that finite-differencetime-domain (FDTD) simulations don't match the data as well as it necessarily should (this behavior is still under investigation), and since the measurement does not cover all the possible zenith angles, a new model has to be built based on the available information

To build such a model, each angular plot (like on the left side of Figure 4) for each frequency is fit to the following: $A \cos ^{2}(B \theta)+C \sin ^{2}(D \theta)$. The $\mathrm{A}, \mathrm{B}, \mathrm{C}$ and $\mathrm{D}$ parameters are then collected for each frequency, and are fit to a 4-degree polynomial. The final model is then the same equation, where the different parameters depend on frequency. The result of this model can be seen in Figure 4, for the VPol antennas, compared to the measurement result and XFDTD ${ }^{1}$ simulations, both in zenith angle-space at one frequency, and in frequency-space at one zenith angle. The developed model is shown to describe the antennas better than the simulations. As a whole, the model describes VPol antenna to about 35\% uncertainty, and a reduced $\chi^{2}$ of 5.3, as opposed to simulation's uncertainty of $70 \%$ and a reduced $\chi^{2}$ of 23.2. For HPol, the model's uncertainty is $40 \%$ with reduced $\chi^{2}$ of 4 , compared to simulation's uncertainty of $85 \%$ and a reduced $\chi^{2}$ of 12.8 .

\footnotetext{
${ }^{1}$ https://www.remcom.com/xfdtd-3d-em-simulation-software/
} 

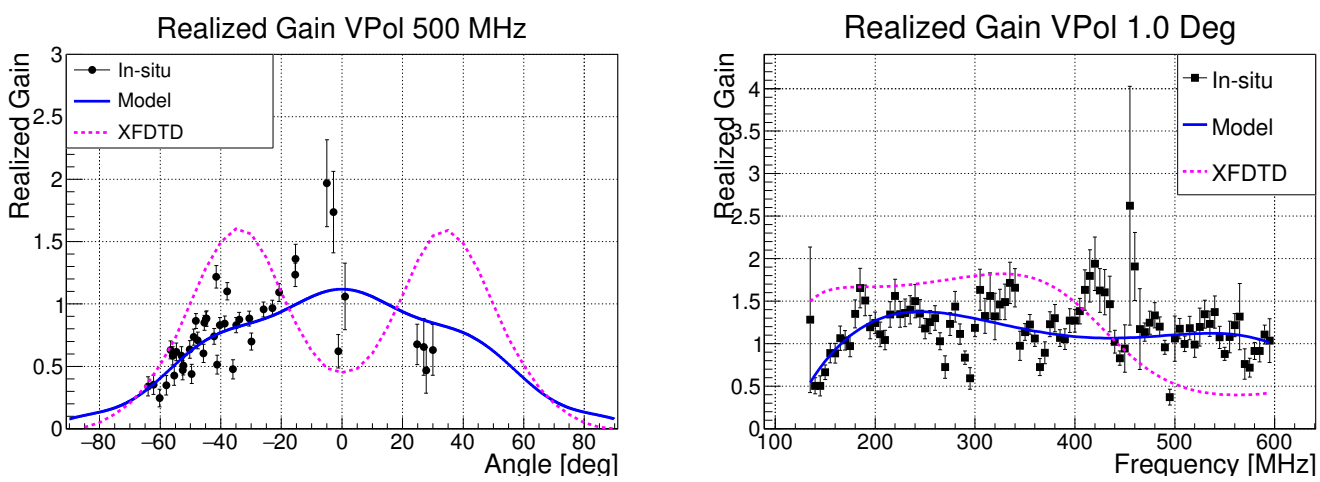

Figure 4. Realized gain as a function of the zenith angle (Left) and to the frequency (Right) to the VPol antenna. This is compared to the XFDTD simulation results as well as the model developed here.

\section{New Simulations}

This antenna model will be used in the new simulation package being developed. As mentioned earlier, these simulations will be following the IceCube modular structure. This will be a new, independent simulation from the one currently in use by ARA (ARASim, [8]), allowing for cross-checking results.

Using the IceCube framework will make it simple to combine this simulation package with IceCube's, which will help to study the occurence of coincidence events with IceCube, or the two detectors' overall sensitivity to GZK neutrinos. Eventually, it will be used to investigate the effect of muon showers on the array, as their stochastic behavior leads to multiple showers along the track, and represent a source of background to the experiment that needs to be understood. Finally, understanding the detector's response to muon showers can be used to confirm the energy scale.

\section{Summary}

With a current configuration of 5 stations, the ARA sensitivity is expected to reach the level of IceCube's. However, this should be confirmed through simulations. A new simulation package using IceCube's modular framework is being developed in that regard. This will use a new antenna model, based on in-situ measurements performed at the South Pole, that describes the antenna's realized gain as a function of zenith angle and frequency. Using that in conjunction with the new station geometry, including the 2 new stations commissioned in January 2018, will then allow to estimate the current sensitivity of the experiment.

\section{References}

[1] K. Greisen, PRL 16, 748 (1966)

[2] G.T. Zatsepin and V.A. Kuzmin, JETP Letters 4, 78 (1966)

[3] V.S. Beresinsky and G.T" Zatsepin, Phys. Lett. B 6, 423 (1969)

[4] G.A. Askaryan, JETP 41, 616 (1962)

[5] G.A. Askaryan, JETP 48, 988 (1965)

[6] P. Allison et al, Astroparticle Physics 35, 457 (2012).

[7] P. Allison et al, Astroparticle Physics 93, 082003 (2016)

[8] P. Allison et al, Astroparticle Physics 70, 62 (2015). 